J. Asiat. Soc. Bangladesh, Sci. 40(2): 295-308, December 2014

\title{
DIVERSITY OF WILDLIFE AT RUHITPUR UNION, KERANIGANJ, DHAKA
}

\author{
M. FIROJ JAMAN', MD. SALAHUDDIN MAJUMDER, MD. SAKHAWAT \\ HOSSAIN, MD. MOKHLESUR RAHMAN AND MAYEN UDDIN \\ Department of Zoology, University of Dhaka, Dhaka-1000, Bangladesh
}

\begin{abstract}
The study was carried out through direct field observation by plot counting; transect line method and interviewing the local people from September 2011 to March 2012. A total of 83 species of wildlife belonging to 18 orders, 34 families and 64 genera was recorded. Of them, $4(4.82 \%)$ species were amphibians, $17(20.48 \%)$ reptiles, 55 $(66.27 \%)$ birds and $7(8.43 \%)$ mammals. Of the amphibians, $3(75 \%)$ were frogs and rest $1(25 \%)$ was toad. Of the reptiles, $3(17.65 \%)$ were turtles, $7(41.18 \%)$ lizards and $7(41.18 \%)$ were snakes. Of the birds, $30(54.55 \%)$ were passerines and remaining 25 $(45.45 \%)$ were non-passerines. Regarding the resident status, $44(80.00 \%)$ species of birds were resident and $11(20.00 \%)$ were winter migrants. Of the mammals, 4 (57.13\%) were rodents, 1 (14.29\%) bats, $1(14.29 \%)$ carnivore and $1(14.29 \%)$ insectivore. The relative abundance showed that $16(20.00 \%)$ were very common, 17 (21.18\%) common, $22(25.88 \%)$ fairly common and 28 (32.94\%) were few. Among the threatened categories, $2(2.35 \%)$ were critically endangered, $3(3.53 \%)$ endangered, $13(15.29 \%)$ vulnerable, $50(61.18 \%)$ lower risk and $15(17.65 \%)$ species were data deficient. Some threats were identified as the cause for the declining of the biodiversity i.e. habitat degradation, climate change, over exploitation of agricultural lands. Implementation of conservation and management practice is necessary to protect the diversity of present species and their population in the studied habitats.
\end{abstract}

Key words: Wildlife diversity, Population density, Habitat, Ruhitpur

\section{Introduction}

Biodiversity encompasses multiple values and is vital for the production of food and to conserve the ecological foundations needed to sustain people's livelihood (Mukul 2007). Distinct physiographic characteristics, variations in hydrological and climatological conditions, and difference in the soil properties in Bangladesh contribute in developing diverse forms of ecosystems enriched with great diversity of flora and fauna (Mittermeier et al. 1998). Due to its unique geo-physical location Bangladesh is exceptionally characterized by a rich biological diversity (Nishat et al. 2002, Hossain 2001 and Barua et al. 2001). Bangladesh has approximately 113 species of mammals, more than 628 species of birds (both passerine and non-passerine), 126 species of reptiles and 22 species of amphibians (Islam 2005). It is widely supposed that the poorest people of those poor countries, who depend most immediately upon local ecosystems for

${ }^{1}$ Corresponding author: Email: mfzaman@yahoo.com 
their livelihoods are somehow responsible for the degradation of biodiversity and will mostly affected by the consequence of this biodiversity loss (CBD 2006 and 2007). Human beings have extensively modified aquatic and terrestrial ecosystems in Bangladesh.

Preparation of base line data on wildlife diversity through scientific study is important to protect them in the study area. Studies had been carried out in different habitats of Bangladesh (Haque 1975, Banerjee 1978, Sarker and Sarker 1983, 1985 and 1988, Rosario and Hai 1996, Jasmin 1996, Hossain and Sarker 1997, Sarker et al. 2001, Jaman et al. 2004, Hossain et al. 2004, Jaman et al. 2011, Rahman et al. 2011 and Rabbi et al. 2011). The present investigation was undertaken to study species composition of wildlife, local status, density of each species and habitat utilization that may help to make conservation plan for wildlife protection in the study area.

\section{Materials and Methods}

Study Area: Ruhitpur union is situated on the bank of Dhaleshwari and Ichamati river in between $23^{\circ} 40^{\prime} 29.12^{\prime \prime}$ north latitude and $90^{\circ} 18^{\prime} 41.14^{\prime \prime}$ east longitude. It is bounded by Kalatia union on the north, Basta union on the east, Serajdikhan upazila on the south, Nawabganj and Singair upazilas on the west (Fig. 1). It is about $12 \mathrm{~km}$ south west from Dhaka city. The total area of the Union is about $15.10 \mathrm{~km}^{2}(3,730$ acres). The study area is mainly covered by cultivated land with a number of ponds, swamps, ditches, canals, beels and homestead areas.

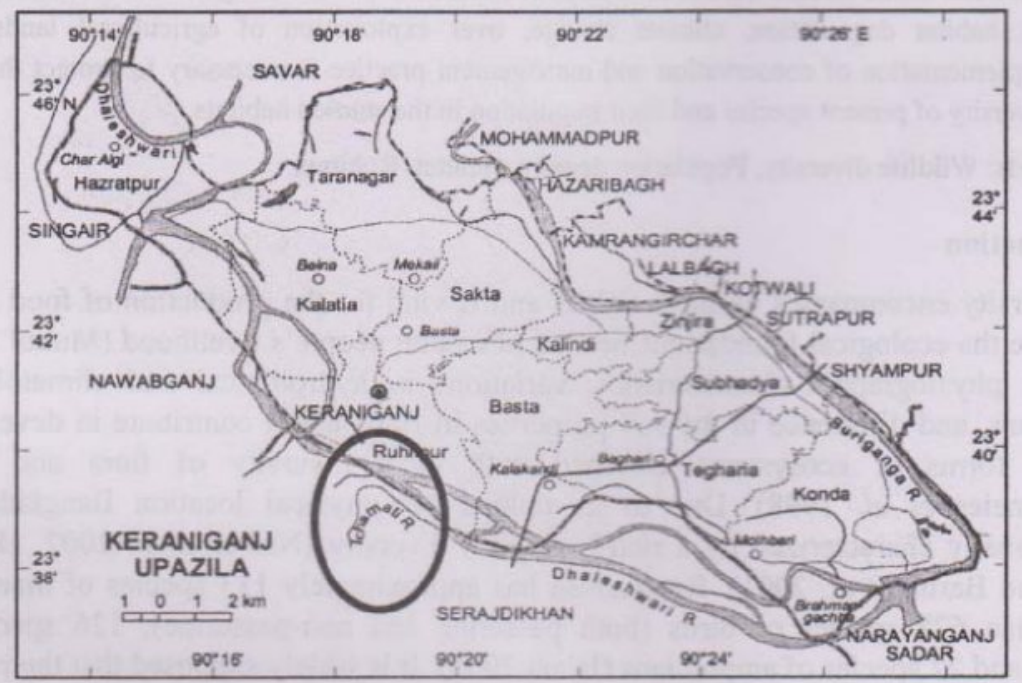

Fig. 1. Study area - map of Keraniganj Upazila. 
There are 16 villages in this union. The villages in Ruhitpur union are lush with vegetation. The backyards of village homes often have dense vegetation, and are particularly important in supporting a number of wildlife. This wildlife use the homestead vegetation and surrounding crop fields (rice, wheat, jute, etc.) for their food and shelter.

The climate of the study area has a moist tropical climate with high rainfall concentrated during the monsoon period from June to October. There were generally four to five months of dry period. The relative humidity remains high, $69 \%-89 \%$ with minor variation. The temperature also remains high with small seasonal differences. Premonsoonal storms were observed from March to May.

The maximum temperature in the year is reached between the first week of April and the end of July. The temperature raises high in February and begins to decrease from October.

The field work for observing and collecting data on wildlife was started in September, 2011 and continued till March, 2012. The observations started in the morning and continued the day long to observe the wild animals including their number, habitats, ecological condition, movement and behavioral activities. The study was based on direct field observation. Observation was made by plot counting and transects line methods. The whole area was divided into 40 plots to observe the amphibians and reptilians fauna. In total 50 transect lines were made for the observation and counting of avifauna. Some avifaunas which were normally hidden in the bush, jungle and branches of trees were recorded by hearing songs and calls. For the mammalian species transect line and plot counting method was used. Some people were interviewed in the study area to collect the data of wild animals especially nocturnal and threatened animals which were not usually observed. Some questionnaires were distributed among the inhabitants of the study area for collecting data on species composition, density and habitat utilizations. During observations, a field binocular was used for watching the animals, a Garmin etrex $\mathrm{H}$ high sensitivity GPS device was used for navigation. Digital camera and maps were also used. Population density was calculated by dividing the total number of each species by the surface area (sq. $\mathrm{km}$ ) where the individual species was counted. The specific method used for the study of different groups of wildlife is as follows:

Amphibians: Plot counting method was used for collecting data on species diversity and population density of amphibians. A total of 40 plots $(100 \mathrm{~m} \times 100 \mathrm{~m})$ was selected in study area. For the identification and taxonomical studies Husain and Rahman (1978) and Khan (2008) were consulted.

Reptiles: In order to survey the lizards and snakes plot counting and transect line method were used. For the identification and taxonomical study Daniel (1983), Sarker and Sarker (1985) and Khan (2008) were consulted.

Birds: Only transect line method ( $500 \mathrm{~m}$ length and $50 \mathrm{~m}$ width) was applied for the census of birds. Ali and Ripley (1983), Hossain and Sarker (1997) and Khan (2008) were consulted for the identification and taxonomical study. 
Mammals: Mammals were studied using transects line and plot counting method. For their identification and taxonomical studies Green (1978), Prater (1993) and Khan (2008) were consulted.

\section{Results And Discussion}

A total of 83 species of wildlife belonging to 18 orders and 34 families was recorded during the study period. Among the total species, 4 were amphibians, 17 reptiles, 55 birds and 7 species were mammals (Fig. 2). Relative abundance showed that 16 species were very common, 17 common, 22 fairly common and 28 were few.

\section{图Amphibia $\square$ Reptiles 1 Birds 回 Mammds}

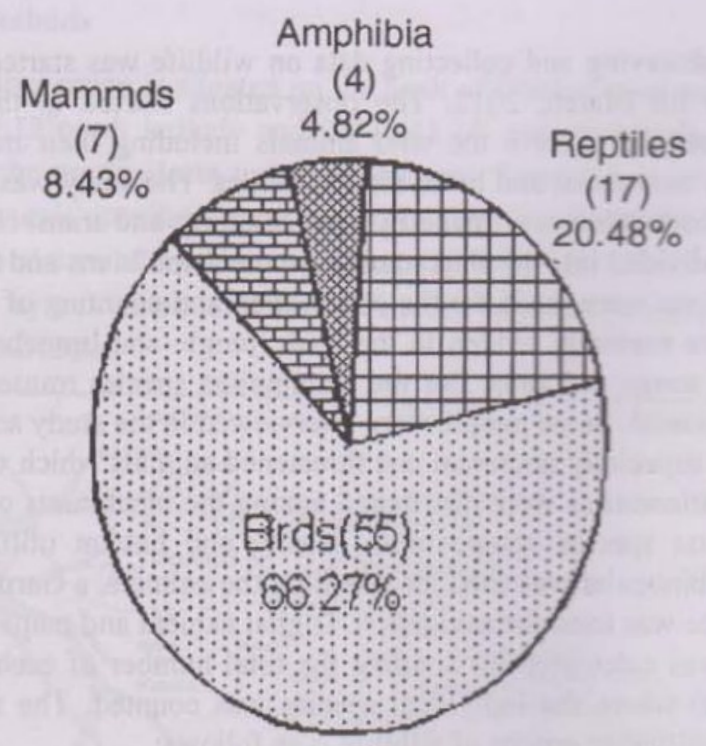

Fig. 2. Wildlife species observed in the study area.

Amphibians: Among the amphibians, $75 \%$ species were frogs and only $25 \%$ was toad. Relative abundance showed that $50 \%$ species were very common and $50 \%$ fairly common (Fig. 3). IUCN threatened categories showed that 3 species were at lower risk and 1 was vulnerable nationally. Population density was the highest in case of Duttaphrynus melanostictus $\left(9.44 / \mathrm{km}^{2}\right)$ followed by Hoplobatrachus tigerinus $\left(9.16 / \mathrm{km}^{2}\right)$ and the lowest in Fejervarya limnocharis $\left(2.77 / \mathrm{km}^{2}\right)$ and Euphlyctis cyanophlyctis $\left(2.77 / \mathrm{km}^{2}\right)$ (Table 1$)$. 


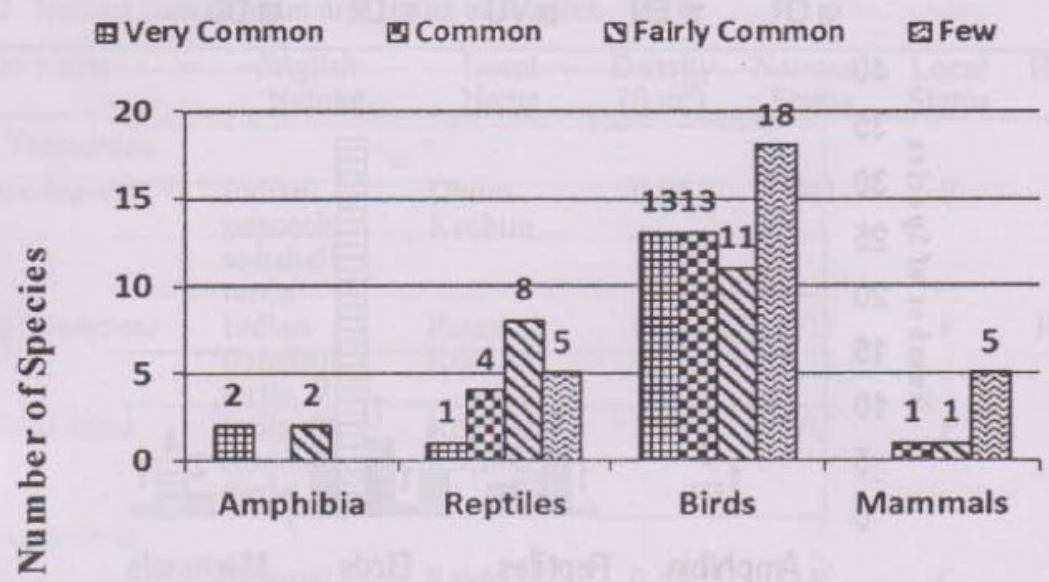

Wildlife species studied

Fig. 3. Relative abundance of recorded wildlife.

Table 1. Species composition and status of amphibians.

\begin{tabular}{llccccc}
\hline Scientific Name & English Name & $\begin{array}{l}\text { Local } \\
\text { Name }\end{array}$ & $\begin{array}{c}\text { Density } \\
\left(\mathrm{km}^{2}\right)\end{array}$ & $\begin{array}{c}\text { National } \\
\text { Status }\end{array}$ & $\begin{array}{c}\text { Local } \\
\text { Status }\end{array}$ & Habitat \\
\hline $\begin{array}{l}\text { Order: Anura } \\
\text { Duttaphrynus } \\
\text { melanostictus }\end{array}$ & $\begin{array}{l}\text { Asian Common } \\
\text { Toad }\end{array}$ & $\begin{array}{l}\text { Kuno } \\
\text { Bang }\end{array}$ & 9.44 & LR & VC & P, h, t \\
$\begin{array}{l}\text { Hoplobatrachus } \\
\text { tigerinus }\end{array}$ & Indian Bull Frog & $\begin{array}{l}\text { Sona } \\
\text { Bang }\end{array}$ & 9.16 & VU & VC & P,e, 1 \\
$\begin{array}{l}\text { Fejervarya } \\
\text { limnocharis }\end{array}$ & Cricket Frog & $\begin{array}{l}\text { Jhijhi } \\
\text { Bang }\end{array}$ & 2.77 & LR & FC & P, e, 1 \\
$\begin{array}{l}\text { Euphlyctis } \\
\text { cyanophlyctis }\end{array}$ & Indian Skipper & $\begin{array}{l}\text { Mali } \\
\text { Bang }\end{array}$ & 2.77 & LR & FC & P,e \\
\hline
\end{tabular}

Reptiles: Among the reptiles, $3(17.65 \%)$ species were turtles, 7 (41.18\%) lizards and 7 $(41.18 \%)$ were snakes. Among the snakes, $3(42.86 \%)$ species were poisonous and remaining $4(57.14 \%)$ non-poisonous. Relative abundance showed that only one $(5.88 \%)$ species was very common, $3(17.65 \%)$ common, $8(47.06 \%)$ fairly common and 5 (29.41\%) few (Fig. 3). IUCN threatened categories showed that 7 species at lower risk, 5 vulnerable, only one endangered and 4 were data deficient (Fig. 4). Population density of Hemidactylus frenatus $\left(8.05 / \mathrm{km}^{2}\right)$ was the highest followed by Hemidactylus brookii $\left(5.27 / \mathrm{km}^{2}\right)$ and Calotes versicolor $\left(5.00 / \mathrm{km}^{2}\right)$ and the lowest in Pangshura tecta $\left(1.11 / \mathrm{km}^{2}\right)$ followed by Lissemys punctata $\left(0.55 / \mathrm{km}^{2}\right)$, Nilssonia hurum $\left(0.55 / \mathrm{km}^{2}\right)$ and Varanus salvator $\left(0.83 / \mathrm{km}^{2}\right)$ (Table 2$)$. 


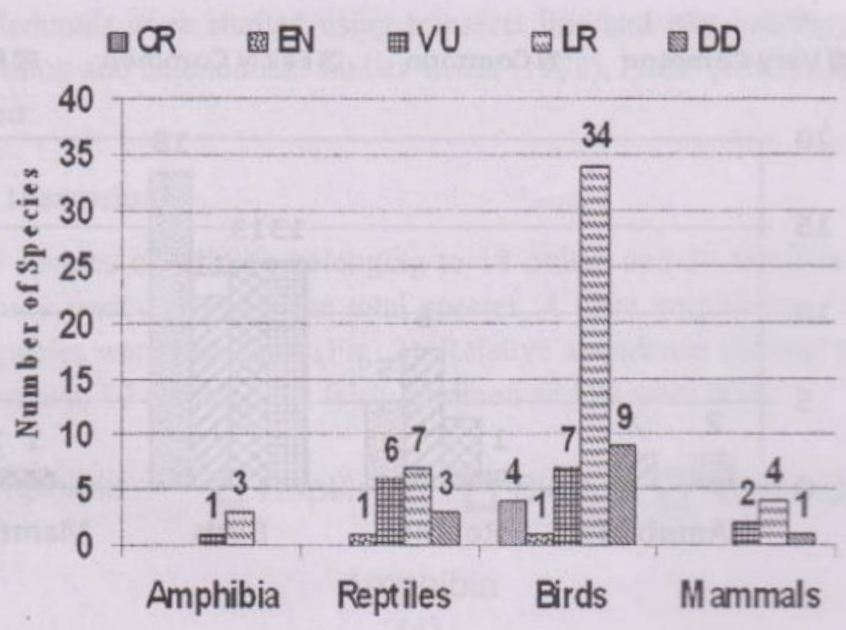

Wildlife species

Fig. 4. Threatened status of recorded wildlife.

Birds: Among the total species of birds, 25 (45.45\%) were non passerines and remaining $30(54.55 \%)$ were passerines. Of the non-passerine birds, $21(84.00 \%)$ were resident and $4(16.00 \%)$ winter migrants. Of the passerine birds, $22(73.33 \%)$ were resident and rest 8 (26.66\%) were winter migrants. Relative abundance showed that $13(23.63 \%)$ species were very common, $13(23.63 \%)$ common, $11(19.30 \%)$ fairly common and $18(31.58 \%)$ were few (Fig. 3). Threatened status showed that 36 species were lower risk, 10 endangered, 6 vulnerable and only 2 were critically endangered (Fig. 4). The highest population density of birds was Acridotheres tristis $\left(245.60 / \mathrm{km}^{2}\right)$ followed by Dicrurus macrocercus $\left(198.00 / \mathrm{km}^{2}\right)$ and Lanius cristatus $\left(100.80 / \mathrm{km}^{2}\right)$ and the lowest was Anas poecilorhyncha $\left(0.80 / \mathrm{km}^{2}\right)$ (Table 3$)$.

Mammals: Of the mammals, one (14.29\%) was shrew, 4 (57.14\%) rodents, one (14.29\%) bat and one (14.29\%) was mongoose. Relative abundance showed that one $(14.29 \%)$ was common, $1(14.29 \%)$ fairly common and $5(71.43 \%)$ were few (Fig. 3). Of the threatened categories, 4 species were at lower risk, only one vulnerable and 2 species were data deficient (Fig. 4). The highest population density was Herpestes edwardsii $\left(4.16 / \mathrm{km}^{2}\right)$ and the lowest was Bandicota bengalensis $\left(0.55 / \mathrm{km}^{2}\right)$ (Table 4). 
Table 2. Species composition and status of reptiles.

\begin{tabular}{|c|c|c|c|c|c|c|}
\hline Sientific Name & $\begin{array}{l}\text { English } \\
\text { Name }\end{array}$ & $\begin{array}{l}\text { Local } \\
\text { Name }\end{array}$ & $\begin{array}{l}\text { Density } \\
\left(\left(\mathrm{km}^{2}\right)\right.\end{array}$ & $\begin{array}{c}\text { National } \\
\text { Status }\end{array}$ & $\begin{array}{l}\text { Local } \\
\text { Status }\end{array}$ & Habitat \\
\hline \multicolumn{7}{|l|}{ Order: Testudines } \\
\hline Nilssonia hurum & $\begin{array}{l}\text { Indian } \\
\text { peacock } \\
\text { softshell } \\
\text { turtle }\end{array}$ & $\begin{array}{l}\text { Dhum } \\
\text { Kachim }\end{array}$ & 0.55 & DD & $\mathrm{F}$ & $\mathrm{P}, \mathrm{p}$ \\
\hline Lissemys punctate & $\begin{array}{l}\text { Indian } \\
\text { flapshell } \\
\text { turtle }\end{array}$ & $\begin{array}{l}\text { Patapori } \\
\text { Kachim }\end{array}$ & 0.55 & VU & $\mathrm{F}$ & $P, c, p$ \\
\hline Pangshura tecta & $\begin{array}{l}\text { Indian } \\
\text { Roofed } \\
\text { turtle }\end{array}$ & $\begin{array}{l}\text { Kori/Hail } \\
\text { Kachim }\end{array}$ & 1.11 & VU & $\mathrm{F}$ & $P, p$ \\
\hline $\begin{array}{l}\text { Order: Lacertelia } \\
\text { Calotes versicolor }\end{array}$ & $\begin{array}{l}\text { Oriental } \\
\text { Garden } \\
\text { Lizard }\end{array}$ & $\begin{array}{l}\text { Rakto } \\
\text { chosa }\end{array}$ & 0.55 & LR & $\mathrm{C}$ & $\mathrm{P}, \mathrm{t}$ \\
\hline Gekko gecko & $\begin{array}{l}\text { Tokyo } \\
\text { Gecko }\end{array}$ & Takkhak & 2.77 & VU & FC & $P, b, x$ \\
\hline $\begin{array}{l}\text { Hemidactylus } \\
\text { frenatus }\end{array}$ & $\begin{array}{l}\text { Common } \\
\text { House } \\
\text { Gecko }\end{array}$ & Tiktiki & 8.05 & LR & VC & $\mathrm{P}, \mathrm{h}, \mathrm{t}$ \\
\hline $\begin{array}{l}\text { Hemidactylus } \\
\text { brookii }\end{array}$ & $\begin{array}{l}\text { Brook's } \\
\text { Gecko }\end{array}$ & Tiktiki & 5.27 & LR & C & $\mathrm{P}, \mathrm{h}$ \\
\hline $\begin{array}{l}\text { Varanus } \\
\text { bengalensis }\end{array}$ & $\begin{array}{l}\text { Bengal } \\
\text { Monitor }\end{array}$ & Gui Shap & 1.38 & EN & FC & $\mathrm{P}, \underset{\mathrm{u}}{\mathrm{d}}, \mathrm{e}$, \\
\hline Mabuya carinata & $\begin{array}{l}\text { Common } \\
\text { Skink }\end{array}$ & Anjan & 3.05 & LR & C & $P, b, 1$ \\
\hline Mabuya dissimilis & $\begin{array}{l}\text { Stripped } \\
\text { Skink }\end{array}$ & Achila & 0.83 & VU & FC & $\mathrm{L}, \mathrm{b}$ \\
\hline \multicolumn{7}{|l|}{ Order: Serpentes } \\
\hline $\begin{array}{l}\text { Ramphotyphlops } \\
\text { braminus }\end{array}$ & $\begin{array}{l}\text { Common } \\
\text { Blind Snake }\end{array}$ & $\begin{array}{l}\text { Dumukha } \\
\text { Shap }\end{array}$ & 2.22 & LR & FC & $\mathrm{L}, \mathrm{h}$ \\
\hline $\begin{array}{l}\text { Xenochrophis } \\
\text { piscator }\end{array}$ & $\begin{array}{l}\text { Checkered } \\
\text { keelback } \\
\text { water snake }\end{array}$ & Dora Shap & 1.38 & LR & FC & $\mathrm{P}, \mathrm{c}$ \\
\hline $\begin{array}{l}\text { Amphiesma } \\
\text { stolatum }\end{array}$ & $\begin{array}{l}\text { Buff } \\
\text { Striped } \\
\text { Keelback }\end{array}$ & Dora Shap & 1.11 & VU & FC & c \\
\hline Ptyas mucosus & $\begin{array}{l}\text { Oriental Rat } \\
\text { Snake }\end{array}$ & $\begin{array}{l}\text { Daraj } \\
\text { Shap }\end{array}$ & 1.11 & LR & FC & $\mathrm{P}, \mathrm{b}$ \\
\hline Enhydris enhydris & $\begin{array}{l}\text { Rainbow } \\
\text { Water- } \\
\text { snake }\end{array}$ & Pani Shap & 1.66 & DD & FC & $\mathrm{P}, \mathrm{c}$ \\
\hline Bungarus fasciatus & $\begin{array}{l}\text { Banded } \\
\text { Krait }\end{array}$ & $\begin{array}{l}\text { Shankhini } \\
\text { Shap }\end{array}$ & 1.11 & VU & $\mathrm{F}$ & $c, d$ \\
\hline Bungarus caeruleus & $\begin{array}{l}\text { Common } \\
\text { Krait }\end{array}$ & Kalkeutey & 0.83 & DD & $\mathrm{F}$ & $c, d$ \\
\hline
\end{tabular}


Table 3. Species composition and status of birds.

\begin{tabular}{|c|c|c|c|c|c|c|}
\hline $\begin{array}{l}\text { Scientific } \\
\text { Name } \\
\end{array}$ & $\begin{array}{l}\text { English } \\
\text { Name } \\
\end{array}$ & Local Name & $\begin{array}{l}\text { Density } \\
\left(/ \mathrm{km}^{2}\right)\end{array}$ & $\begin{array}{l}\text { Nationa } \\
\text { I Status }\end{array}$ & $\begin{array}{l}\text { Local } \\
\text { Status }\end{array}$ & Habitat \\
\hline \multicolumn{7}{|c|}{ Order: Pelecaniformes } \\
\hline $\begin{array}{l}\text { Phalacrocorax } \\
\text { niger }\end{array}$ & $\begin{array}{l}\text { Little } \\
\text { Cormorant }\end{array}$ & $\begin{array}{l}\text { Choto } \\
\text { Pankouri }\end{array}$ & 3.60 & VU & FC & $\mathrm{P}, \mathrm{a}, \mathrm{c}, \mathrm{e}$ \\
\hline \multicolumn{7}{|c|}{ Order: Ciconiformes } \\
\hline Ardeola grayii & Pond Heron & Kani Bok & 21.60 & LR & VC & $\mathrm{P}, \mathrm{a}, \mathrm{d}, \mathrm{e}$ \\
\hline Bubulcus ibis & Cattle Egret & Go Bok & 8.40 & LR & $\mathrm{C}$ & $P, c, d, e$ \\
\hline Egretta alba & Large Egret & Baro Bok & 3.20 & VU & $\mathrm{F}$ & $P, c, d, e$ \\
\hline $\begin{array}{l}\text { Egretta } \\
\text { intermedia }\end{array}$ & $\begin{array}{l}\text { Intermediate } \\
\text { Egret }\end{array}$ & Majhari Bok & 3.60 & VU & $\mathrm{FC}$ & $\mathrm{P}, \mathrm{c}, \mathrm{d}, \mathrm{e}$ \\
\hline $\begin{array}{l}\text { Egretta } \\
\text { garzetta }\end{array}$ & Little Egret & Choto Bok & 3.20 & LR & F & $\mathrm{P}, \mathrm{c}, \mathrm{d}, \mathrm{e}$ \\
\hline \multicolumn{7}{|c|}{ Order: Anseriformes } \\
\hline $\begin{array}{l}\text { Dendrocygna } \\
\text { javanica }\end{array}$ & $\begin{array}{l}\text { Lesser } \\
\text { Whistling- } \\
\text { Duck }\end{array}$ & $\begin{array}{l}\text { Choto Sarali } \\
\text { Hans }\end{array}$ & 3.60 & VU & FC & $\mathrm{P}, \mathrm{a}, \mathrm{e}$ \\
\hline Anas acuta & $\begin{array}{l}\text { Northern } \\
\text { Pintail }\end{array}$ & Lenja Hans & 4.80 & DD & $\mathrm{C}$ & $\mathrm{P}, \mathrm{c}, \mathrm{e}$ \\
\hline $\begin{array}{l}\text { Anas } \\
\text { poecilorhyncha }\end{array}$ & $\begin{array}{l}\text { Spot-billed } \\
\text { Duck }\end{array}$ & $\begin{array}{l}\text { Pati/ } \\
\text { Mete Hans }\end{array}$ & 0.80 & CR & $\mathrm{F}$ & $P, a, c, e$ \\
\hline \multicolumn{7}{|c|}{ Order: Columbiformes } \\
\hline Columba livia & $\begin{array}{l}\text { Blue Rock } \\
\text { Pigeon }\end{array}$ & Jalali Kabutor & 15.60 & LR & VC & $\mathrm{P}, \mathrm{a}, \mathrm{h}$ \\
\hline $\begin{array}{l}\text { Streptopelia } \\
\text { chinensis }\end{array}$ & $\begin{array}{l}\text { Spotted } \\
\text { Dove }\end{array}$ & Tila Ghugu & 12.00 & EN & VC & $\begin{array}{c}\mathrm{P}, \mathrm{a}, \mathrm{b} \\
\mathrm{d}, \mathrm{t}\end{array}$ \\
\hline $\begin{array}{l}\text { Streptopelia } \\
\text { decaocto }\end{array}$ & Ring Dove & Raj Ghughu & 4.40 & $\mathrm{CR}$ & $\mathrm{F}$ & $\begin{array}{c}\mathrm{P}, \mathrm{a}, \mathrm{d}, \\
\mathrm{l}, \mathrm{t}\end{array}$ \\
\hline $\begin{array}{l}\text { Chalcophaps } \\
\text { indica }\end{array}$ & $\begin{array}{l}\text { Common } \\
\text { Emerald } \\
\text { Dove }\end{array}$ & $\begin{array}{l}\text { Sabuj/ } \\
\text { Raj Ghughu }\end{array}$ & 2.40 & VU & $\mathrm{F}$ & $\mathrm{P}, \mathrm{h}, \mathrm{t}$ \\
\hline \multicolumn{7}{|c|}{ Order: Cuculiformes } \\
\hline $\begin{array}{l}\text { Eudynamys } \\
\text { scolopacea }\end{array}$ & Asian Koel & Kokil & 6.80 & LR & $\mathrm{C}$ & $P, t$ \\
\hline $\begin{array}{l}\text { Cacomantis } \\
\text { merulinus }\end{array}$ & $\begin{array}{l}\text { Plaintive } \\
\text { Cuckoo }\end{array}$ & Sorgom & 5.60 & LR & FC & $\mathrm{P}, 1$ \\
\hline $\begin{array}{l}\text { Hierococcyx } \\
\text { varius }\end{array}$ & $\begin{array}{l}\text { Common } \\
\text { Hawk Cuckoo }\end{array}$ & Chokh gelo & 1.20 & DD & $\mathrm{F}$ & $b, 1, x$ \\
\hline $\begin{array}{l}\text { Centropus } \\
\text { sinensis }\end{array}$ & $\begin{array}{l}\text { Greater } \\
\text { Coucul }\end{array}$ & $\begin{array}{l}\text { Baro Kana } \\
\text { Kukka }\end{array}$ & 6.00 & VU & $\mathrm{C}$ & $\mathrm{P}, \mathrm{l}, \mathrm{t}$ \\
\hline
\end{tabular}


Diversity of wildlife at Ruhitpur union

Contd.

\begin{tabular}{|c|c|c|c|c|c|c|}
\hline $\begin{array}{l}\text { Scientific } \\
\text { Name }\end{array}$ & $\begin{array}{l}\text { English } \\
\text { Name }\end{array}$ & Local Name & $\begin{array}{l}\text { Density } \\
\left(/ \mathrm{km}^{2}\right)\end{array}$ & $\begin{array}{l}\text { Nationa } \\
1 \text { Status }\end{array}$ & $\begin{array}{l}\text { Local } \\
\text { Status }\end{array}$ & Habitat \\
\hline \multicolumn{7}{|c|}{ Order: Coraciformes } \\
\hline Alcedo atthis & $\begin{array}{l}\text { Common } \\
\text { Kingfisher }\end{array}$ & $\begin{array}{l}\text { Khude } \\
\text { Machranga }\end{array}$ & 7.20 & LR & $\mathrm{C}$ & $\mathrm{P}, \mathrm{e}$ \\
\hline $\begin{array}{l}\text { Halcyon } \\
\text { smyrnensis }\end{array}$ & $\begin{array}{l}\text { White } \\
\text { breasted } \\
\text { Kingfisher }\end{array}$ & $\begin{array}{l}\text { Shadabuk } \\
\text { Machranga }\end{array}$ & 5.20 & LR & FC & $P, e, t$ \\
\hline $\begin{array}{l}\text { Merops } \\
\text { orientalis }\end{array}$ & $\begin{array}{l}\text { Green Bee } \\
\text { Eater }\end{array}$ & $\begin{array}{l}\text { Suichora/ } \\
\text { Bashpoti }\end{array}$ & 27.6 & DD & VC & $\begin{array}{c}P, a, b, \\
\text { e }\end{array}$ \\
\hline $\begin{array}{l}\text { Merops } \\
\text { leschenaulti }\end{array}$ & $\begin{array}{l}\text { Chestnut } \\
\text { Headed Bee } \\
\text { Eater }\end{array}$ & - & 10.80 & VU & C & $\begin{array}{c}\mathrm{P}, \mathrm{a}, \mathrm{b}, \\
\mathrm{e}\end{array}$ \\
\hline \multicolumn{7}{|c|}{ Order: Passeriformes } \\
\hline $\begin{array}{l}\text { Passer } \\
\text { domesticus }\end{array}$ & $\begin{array}{l}\text { House } \\
\text { Sparrow }\end{array}$ & Charui & 26.80 & LR & VC & $\mathrm{P}, \mathrm{a}, \mathrm{h}$, \\
\hline $\begin{array}{l}\text { Anthus } \\
\text { campestris }\end{array}$ & Tawny Pipit & - & 5.20 & LR & FC & $P, a, b$ \\
\hline $\begin{array}{l}\text { Anthus } \\
\text { hodgsoni }\end{array}$ & $\begin{array}{l}\text { Olive- } \\
\text { backed Pipit }\end{array}$ & - & 5.60 & DD & FC & $\mathrm{P}, \mathrm{a}, \mathrm{b}$ \\
\hline Anthus rufulus & $\begin{array}{l}\text { Paddy-field } \\
\text { Pipit }\end{array}$ & - & 54.00 & LR & VC & $\begin{array}{c}P, a, b \\
d\end{array}$ \\
\hline $\begin{array}{l}\text { Motacilla } \\
\text { citreola }\end{array}$ & $\begin{array}{l}\text { Citrine } \\
\text { Wagtail }\end{array}$ & - & 5.60 & DD & $\mathrm{F}$ & $\mathrm{P}, \mathrm{b}, \mathrm{d}, \mathrm{t}$ \\
\hline $\begin{array}{l}\text { Dicrurus } \\
\text { macrocercus }\end{array}$ & $\begin{array}{l}\text { Black } \\
\text { Drongo }\end{array}$ & Kalo Fingey & 198.00 & LR & VC & $\begin{array}{l}\mathrm{P}, \mathrm{a}, \mathrm{b} \\
\mathrm{d}, \mathrm{e}, \mathrm{l}\end{array}$ \\
\hline $\begin{array}{l}\text { Dicrurus } \\
\text { aeneus }\end{array}$ & $\begin{array}{l}\text { Bronzed } \\
\text { Drongo }\end{array}$ & $\begin{array}{l}\text { Dhusor } \\
\text { Fingey }\end{array}$ & 24.80 & LR & VC & $\begin{array}{c}\mathrm{t}, \mathrm{x}, \mathrm{y} \\
\mathrm{P}, \mathrm{a}, \mathrm{d} \\
\mathrm{l}, \mathrm{t}\end{array}$ \\
\hline Sturnus contra & $\begin{array}{l}\text { Pied } \\
\text { Starling }\end{array}$ & $\begin{array}{l}\text { Gobrey } \\
\text { Shalik }\end{array}$ & 8.40 & LR & $\mathrm{C}$ & $P, a, d, t$ \\
\hline $\begin{array}{l}\text { Sturnus } \\
\text { malabaricus }\end{array}$ & $\begin{array}{l}\text { Chestnut- } \\
\text { tailed } \\
\text { Starling }\end{array}$ & Shalik & 2.80 & LR & $\mathrm{F}$ & $P, t$ \\
\hline $\begin{array}{l}\text { Acridotheres } \\
\text { tristis }\end{array}$ & $\begin{array}{l}\text { Common } \\
\text { Myna }\end{array}$ & Bhat Shalik & 245.60 & LR & VC & $\begin{array}{l}\mathrm{P}, \mathrm{a}, \mathrm{b} \\
\mathrm{d}, \mathrm{1}, \mathrm{t}\end{array}$ \\
\hline $\begin{array}{l}\text { Acridotheres } \\
\text { fuscus }\end{array}$ & $\begin{array}{l}\text { Jungle } \\
\text { Myna }\end{array}$ & Jhuti Shalik & 10.40 & LR & $\mathrm{C}$ & $\begin{array}{c}x, y \\
P, a, b \\
d, l, t\end{array}$ \\
\hline $\begin{array}{l}\text { Corvus } \\
\text { splendens }\end{array}$ & House Crow & Pati Kak & 92.80 & LR & VC & $\begin{array}{c}x, y \\
\mathrm{P}, \mathrm{a}, \mathrm{h}\end{array}$ \\
\hline $\begin{array}{l}\text { Corvus } \\
\text { macrorhynchos }\end{array}$ & Jungle Crow & Dar Kak & 8.00 & LR & FC & $\mathrm{P}, \underset{\mathrm{t}}{\mathrm{a}}, \mathrm{h}$ \\
\hline $\begin{array}{l}\text { Dendrocitta } \\
\text { vagabunda }\end{array}$ & $\begin{array}{l}\text { Rufos Tree- } \\
\text { pie }\end{array}$ & Harichacha & 10.00 & LR & $\mathrm{C}$ & $\begin{array}{c}\mathrm{P}, \mathrm{l}, \mathrm{t} \\
\mathrm{x}\end{array}$ \\
\hline
\end{tabular}


Contd.

\begin{tabular}{|c|c|c|c|c|c|c|}
\hline $\begin{array}{l}\text { Scientific } \\
\text { Name }\end{array}$ & $\begin{array}{l}\text { English } \\
\text { Name }\end{array}$ & Local Name & $\begin{array}{l}\text { Density } \\
\left(/ \mathrm{km}^{2}\right)\end{array}$ & $\begin{array}{l}\text { Nationa } \\
\text { I Status }\end{array}$ & $\begin{array}{l}\text { Local } \\
\text { Status }\end{array}$ & Habitat \\
\hline Aegithina & Common & Fatik Jal & 5.20 & LR & $\mathrm{FC}$ & $\mathrm{b}, 1$ \\
\hline tiphia & Iora & & & & & \\
\hline Oriolus oriolus & $\begin{array}{l}\text { Golden } \\
\text { Oriole }\end{array}$ & Bene Bou & 8.40 & LR & FC & $\begin{array}{c}P, a, b \\
t\end{array}$ \\
\hline $\begin{array}{l}\text { Oriolus } \\
\text { xanthornus }\end{array}$ & $\begin{array}{l}\text { Black } \\
\text { Headed } \\
\text { Oriole }\end{array}$ & Haldey Pakhi & 7.60 & LR & FC & $\begin{array}{l}\mathrm{P}, \mathrm{a}, \mathrm{b} \\
\mathrm{l}, \mathrm{x}, \mathrm{y}\end{array}$ \\
\hline $\begin{array}{l}\text { Pycnonotus } \\
\text { cafer }\end{array}$ & $\begin{array}{l}\text { Red Vented } \\
\text { Bulbul }\end{array}$ & Bulbuli & 8.80 & LR & C & $P, t, x, y$ \\
\hline Pycnonotus & Red & Shipahi & 3.60 & $\mathrm{CR}$ & $\mathrm{F}$ & $b, t$ \\
\hline jocosus & $\begin{array}{l}\text { Whiskered } \\
\text { Bulbul }\end{array}$ & Bulbuli & & & & \\
\hline $\begin{array}{l}\text { Orthotomus } \\
\text { sutorius }\end{array}$ & $\begin{array}{l}\text { Common } \\
\text { Tailor Bird }\end{array}$ & Tuntuni & 23.60 & LR & VC & $\begin{array}{l}P, b, h \text {, } \\
t, x, y\end{array}$ \\
\hline $\begin{array}{l}\text { Phylloscopus } \\
\text { fuscatus }\end{array}$ & $\begin{array}{l}\text { Dusky } \\
\text { Warbler }\end{array}$ & a - & 3.20 & LR & $\mathrm{F}$ & P \\
\hline $\begin{array}{l}\text { Phylloscopus } \\
\text { reguloides }\end{array}$ & $\begin{array}{l}\text { Blyth's Leaf } \\
\text { Warbler }\end{array}$ & हरे & 2.00 & DD & F & $P$ \\
\hline $\begin{array}{l}\text { Acrocephalus } \\
\text { stentoreus }\end{array}$ & $\begin{array}{l}\text { Clamorous } \\
\text { Reed } \\
\text { Warbler }\end{array}$ & Tikra & 2.80 & LR & $\mathrm{F}$ & $\mathrm{P}, \mathrm{l}, \mathrm{t}$ \\
\hline $\begin{array}{l}\text { Mirafra } \\
\text { assamica }\end{array}$ & $\begin{array}{l}\text { Rufous- } \\
\text { winged } \\
\text { Bush Lark }\end{array}$ & Bharat & 6.00 & LR & F & $\mathrm{P}, \mathrm{b}$ \\
\hline $\begin{array}{l}\text { Calandrella } \\
\text { raytal }\end{array}$ & Sand Lark & Dhulcharai & 43.20 & LR & VC & P, d, l, \\
\hline $\begin{array}{l}\text { Lanius } \\
\text { cristatus }\end{array}$ & $\begin{array}{l}\text { Brown } \\
\text { Shrike }\end{array}$ & Badami Kosai & 100.80 & DD & VC & $\begin{array}{r}\mathrm{P}, \mathrm{a}, \mathrm{b}, \\
\mathrm{d}, \mathrm{l}, \mathrm{t}\end{array}$ \\
\hline Lanius schach & $\begin{array}{l}\text { Rufous- } \\
\text { backed } \\
\text { Shrike }\end{array}$ & $\begin{array}{l}\text { Dabra/Baghat } \\
\text { iki }\end{array}$ & 5.20 & LR & F & $\begin{array}{r}\mathrm{P}, \mathrm{a}, \mathrm{b}, \\
\mathrm{d}, \mathrm{l}, \mathrm{t}\end{array}$ \\
\hline $\begin{array}{l}\text { Copsychus } \\
\text { saularis }\end{array}$ & $\begin{array}{l}\text { Oriental } \\
\text { Magpie } \\
\text { Robin }\end{array}$ & Doel & 8.00 & LR & C & $\begin{array}{r}\mathrm{P}, \mathrm{a}, \mathrm{b}, \\
\mathrm{h}, \mathrm{l}, \mathrm{t}\end{array}$ \\
\hline \multicolumn{7}{|c|}{ Order: Piciformes } \\
\hline $\begin{array}{l}\text { Dinopium } \\
\text { benghalense }\end{array}$ & $\begin{array}{l}\text { Black- } \\
\text { rumped } \\
\text { Flameback }\end{array}$ & Kath thokra & 8.40 & LR & C & $\mathrm{P}, \mathrm{t}$ \\
\hline $\begin{array}{l}\text { Dendrocopos } \\
\text { macei }\end{array}$ & $\begin{array}{l}\text { Fulvous- } \\
\text { breasted } \\
\text { Woodpecker }\end{array}$ & Kath thokra & 5.60 & LR & $\mathrm{F}$ & $\mathrm{P}, \mathrm{t}$ \\
\hline \multicolumn{7}{|c|}{ Order: Charadriiformes } \\
\hline $\begin{array}{l}\text { Actitis } \\
\text { hypoleucos }\end{array}$ & $\begin{array}{l}\text { Common } \\
\text { Sandpiper }\end{array}$ & - & 9.20 & DD & C & $P, d, e$ \\
\hline
\end{tabular}


Contd.

\begin{tabular}{llrrrrr}
\hline $\begin{array}{l}\text { Scientific } \\
\text { Name }\end{array}$ & $\begin{array}{l}\text { English } \\
\text { Name }\end{array}$ & Local Name & $\begin{array}{c}\text { Density } \\
\left(/ \mathrm{km}^{2}\right)\end{array}$ & $\begin{array}{l}\text { Nationa } \\
\text { I Status }\end{array}$ & $\begin{array}{l}\text { Local } \\
\text { Status }\end{array}$ & Habitat \\
\hline $\begin{array}{l}\text { Calidris } \\
\text { ferruginea }\end{array}$ & $\begin{array}{l}\text { Curlew } \\
\text { Order: Falconiformes } \\
\begin{array}{l}\text { Milvus } \\
\text { migrans }\end{array}\end{array}$ & Black Kite & 5.60 & DD & F & P, d, e \\
$\begin{array}{l}\text { Haliastur } \\
\text { indus }\end{array}$ & $\begin{array}{l}\text { Brahminy } \\
\text { Kite }\end{array}$ & - & 4.00 & CR & F & P, a, b, t \\
\hline
\end{tabular}

Table 4. Status of Mammals.

\begin{tabular}{|c|c|c|c|c|c|c|}
\hline $\begin{array}{l}\text { Scientific } \\
\text { Name }\end{array}$ & English Name & $\begin{array}{l}\text { Local } \\
\text { Name }\end{array}$ & $\begin{array}{l}\text { Density } \\
\left(/ \mathrm{km}^{2}\right)\end{array}$ & $\begin{array}{c}\text { National } \\
\text { Status }\end{array}$ & $\begin{array}{l}\text { Local } \\
\text { status }\end{array}$ & Habitat \\
\hline \multicolumn{7}{|c|}{ Order: Soricomorpha } \\
\hline $\begin{array}{l}\text { Suncus } \\
\text { murinus }\end{array}$ & $\begin{array}{l}\text { Asian House } \\
\text { Shrew }\end{array}$ & $\begin{array}{l}\text { Chika/ } \\
\text { Chhucho }\end{array}$ & 2.22 & LR & FC & $\mathrm{P}, \mathrm{b}, \mathrm{h}$ \\
\hline \multicolumn{7}{|c|}{ Order: Rodentia } \\
\hline Mus musculus & House mouse & Nengti Idur & 1.11 & LR & $\mathrm{F}$ & $\mathrm{P}, \mathrm{h}$ \\
\hline $\begin{array}{l}\text { Vandeleuria } \\
\text { oleracea }\end{array}$ & $\begin{array}{l}\text { Asiatic Long- } \\
\text { tailed Climbing } \\
\text { Mouse }\end{array}$ & Gecho Idur & 0.83 & DD & F & $\mathrm{P}, \mathrm{d}, \mathrm{h}$ \\
\hline $\begin{array}{l}\text { Bandicota } \\
\text { indica }\end{array}$ & $\begin{array}{l}\text { Greater Bandicot } \\
\text { Rat }\end{array}$ & $\begin{array}{l}\text { Dhari/Baro } \\
\text { Idur }\end{array}$ & 0.83 & LR & $\mathrm{F}$ & $P, \underset{l}{d}, h$, \\
\hline $\begin{array}{l}\text { Bandicota } \\
\text { bengalensis }\end{array}$ & $\begin{array}{l}\text { Lesser Bandicot } \\
\text { Rat }\end{array}$ & $\begin{array}{l}\text { Dhari/Baro } \\
\text { Idur }\end{array}$ & 0.55 & LR & $\mathrm{F}$ & P, d, h, l \\
\hline \multicolumn{7}{|c|}{ Order: Chiroptera } \\
\hline $\begin{array}{l}\text { Pteropus } \\
\text { giganteus }\end{array}$ & $\begin{array}{l}\text { Indian Flying- } \\
\text { fox }\end{array}$ & $\begin{array}{l}\text { Baro } \\
\text { Badur }\end{array}$ & 0.83 & VU & $\mathrm{F}$ & $\mathrm{a}, \mathrm{t}$ \\
\hline \multicolumn{7}{|c|}{ Order: Carnivora } \\
\hline $\begin{array}{l}\text { Herpestes } \\
\text { edwardsii }\end{array}$ & $\begin{array}{l}\text { Indian Gray } \\
\text { Mongoose }\end{array}$ & Baro Benji & 4.16 & VU & C & $\begin{array}{c}P, b, 1 \\
y\end{array}$ \\
\hline
\end{tabular}

Note; $\mathrm{VC}=$ Very Common; $\mathrm{C}=$ Common; $\mathrm{FC}=$ Fairly Common; $\mathrm{F}=$ Few; $\mathrm{CR}=$ Critically Endangered; $\mathrm{EN}=$ Endangered $\mathrm{VU}=$ Vulnerable; $\mathrm{LR}=$ Lower Risk; $\mathrm{DD}=$ Data Deficient; Habitat: $\mathrm{p}=$ Plain land; $\mathrm{a}=$ flying in the air; $\mathrm{b}=$ bushes and scrubs; $\mathrm{d}=$ cultivated land; $\mathrm{e}=$ water edge; $\mathrm{h}=$ houses in the plain land; $\mathrm{l}=$ open land in the plain land; $\mathrm{t}=$ trees in the plain land; $\mathrm{x}=$ road side; $\mathrm{y}=$ grave yard.

From the above results and discussion it may be mentioned that some factors are affecting biodiversity of Ruhitpur union such as (1) Habitat degradation due to the increase of human population, (2) Extending of agricultural land and increase of land use in the above mentioned habitats, (3) Wide scale destruction of the natural habitat where wild animals are inhabiting, (4) Illegal hunting, trapping and collecting of young birds and mammals from their nests causing depletion of wildlife population from the study 
area. (5) Agrochemicals are being randomly used by the farmers without having proper knowledge, thus adding pollutants to the environment of wildlife and (6) Ignorance of local people about the importance of the biodiversity and conservation of wildlife species.

To protect wildlife species, habitat degradation should be prevented, so that the habitats occupied by the wildlife cannot be hampered by the human population. Killing, hunting or trapping of wildlife must be stopped for the survival of threatened (critically endangered, endangered and vulnerable) wildlife. Creation of public awareness through organizing public programs, like discussion in the educational institutions and stakeholders, etc. and distribution of leaf-lets, hand notes, etc. may help to crease consciousness about the importance of the biodiversity.

\section{Acknowledgement}

Authors are grateful to the volunteers and local administrations of Keraniganj upazila for their help, cooperation and continuous supports.

\section{References}

Ali, S. and S.D. Ripley. 1983. A pictorial guide to the birds of Indian Sub-continent. Bombay Nat. Hist. Soc. Bombay, India. 177 pp.

Banerjee, R.K. 1978. Status and composition of avifauna of the Curzon Hall campus and Ramna Park, Dhaka. M.Sc. thesis. Department of Zoology, University of Dhaka, Bangladesh.

Barua, S.P., M.M.H. Khan and A.H.M.A. Reza. 2001. The Status of Alien Invasive Species in Bangladesh and their Impact on the Ecosystems. In: Alien Invasive Species-Report of workshop on Alien Invasive Species (ed P. Balakrishna). IUCN Regional Biodiversity Programme of Asia, Colombo, Sri Lanka. 1-7 pp.

CBD. 2006. Global Biodiversity Outlook 2. Secretariat of the Convention on Biological Diversity (SCBD), Montreal. 81 pp.

CBD. 2007. Biodiversity and Climate Change. Secretariat of the Convention on Biological Diversity, Montreal. 48 pp.

Daniel, J.C. 1983. The book of Indian Reptiles. India, Bombay Nat. Hist. Soc. pp. 143.

Encyclopedia of Flora and Fauna of Bangladesh. 2009. Asiatic Society of Bangladesh. 1-28.

Green, K.M. 1978. Primates of Bangladesh. A preliminary Survey of Population and Habitat. Biological conservation. 13(2): 11-160

Haque, M. N. 1975. The avifauna of Madhupur Forest (With notes on ecology, status, distribution and food.) M.Sc. thesis. Department of Zoology, University of Dhaka, Bangladesh.

Hossain, M.K. 2001. Overview of the forest biodiversity in Bangladesh. In: Assessment, conservation and sustainable use of forest biodiversity (CBD Technical Series no.3). Secretariat of the Convention on Biological Diversity, Montreal, 33-35 pp. 
Hossain, M.L. and S.U. Sarker. 1997. Birds of Hatiya Island, Noakhali, Bangladesh. Dhaka Univ. J. Biol. Sci., 6(1):39-48.

Hossain, M.L., M.F Jaman and S.U. Sarkar. 2004. Diversity of Herpeto-Mammalian fauna and their conservation issues in Hatiya Island, Bangladesh, Tropical Biodiversity, 8(2): 7178.

Husain, K.Z. and M.M. Rahman. 1978. The Amphibian fauna of Bangladesh. Bangladesh J. of Zoology. 6(2): 157-158.

Islam, T.U. 2005. Third Bangladesh National Report Submitted to Secretariat of Convention on Biological Diversity. Ministry of Environment and Forest, Bangladesh Secretariat, Dhaka. 153 pp.

Jaman, M.F., M.S. Rahman and M.E. Haque. 2011. Diversity of avifauna at the Bangladesh Academy for Rural Development (BARD), Kotbari, Comilla. Univ. J. Zool. Rajshahi Univ. 30: 41-44.

Jaman, M.F., M.S. Zahedul and S.U. Sarker. 2004. Ecology, conservation problems and status of avifauna of Noakhali Charbata coastal area. J. NOAMI. 21(1): 1-13.

Jasmin, H. 1996. The wildlife fauna of Bangladesh Rifles Head Quarter, Dhaka. M.Sc. thesis (unpublished) Department of Zoology, University of Dhaka, Bangladesh.

Khan, M.M.H. 2008. Protected Areas of Bangladesh-A. Guide to Wildlife. Nishorgo Support Program, Bangladesh. Forest Department. 304 pp.

Koziell, I. 2001. Diversity not Adversity: Sustaining Livelihoods with Biodiversity. International Institute for Environment and Development (IIED) and Department for International Development (DFID), England. 58 pp.

Mittermeier, R.A., N. Myers, J.B. Thomsen, G.A. Da Fonseca and S.Olivieri. 1998. Biodiversity hotspots and major tropical wilderness areas: approaches to setting conservation priorities. Conservation Biology. 12: 516-520.

Mukul, S.A. 2007. Biodiversity Conservation Strategies in Bangladesh: The State of Protected Areas. Tigerpaper. 34(3): 28-32.

Nishat, A., S.M.I. Huq, S.P. Barua, A.H.M.A. Reza and A.S.M. Khan. 2002. Bio-ecological Zones of Bangladesh. The World Conservation Union (IUCN), Bangladesh, 141 pp.

Prater, S.H. 1993. The book of Indian animals Bombay: Bombay Nat. Hist. Soc. xi +324 pp.

Rabbi, M.G., S.U. Sarker and M.F. Jaman. 2011. Ecology and status of avifauna of Nijhum Dwip and Damar Char, Noakhali and the conservation issues. J. NOAMI. 28(2): 59-71.

Rahman, M.S., S.U. Sarker and M.F. Jaman. 2011. Ecological status of the herpeto-mammalian fauna of the Padma river and its adjacent areas, Rajshahi and their conservation issues. $J$. NOAMI. 28(1): 49-61.

Rosario, E.A. and S.K. Hai. 1996. List of amphibians, reptiles, birds and mammals in the Sundarbans natural mangrove Stand and adjacent Coastal areas. Forest resources management project. Mandala Agricultural Development Corporation (MADECOR). Dhaka, Bangladesh. 126-135 pp.

Sarker, S.U. and N.J. Sarker. 1983. Endangered wildlife of Bangladesh. Tigerpaper. 10(2): 26-28. 
Sarker, S.U. and N.J. Sarker. 1985. Reptiles of Bangladesh, TigerPaper. 12(2): 6-12.

Sarker, S.U. and N.J. Sarker. 1988. Wildlife of Bangladesh. A systematic List. Nilkhet Babupura, Dhaka: The Rico Printer. 59pp.

Sarker, S.U., M.F. Jaman, M.L. Hossain and N.J. Sarker. 2001. Wildlife diversity of Maheskhali Island: their Ecology and Conservation Issues. J. NOAMI. 18(1): 17-31.

(Received revised manuscript on 6 April 2014) 